\title{
The effects of spiral arms on the multi-phase ISM
}

\author{
Anvar Shukurov \& Graeme R. Sarson \\ School of Mathematics $\&$ Statistics, University of Newcastle, Newcastle NE1 7RU, \\ U.K.
}

Åke Nordlund

NBIfAFG \& TAC, Juliane Maries Vej 30, DK-2100 Copenhagen Ø, Denmark

Boris Gudiksen

Inst. Solar Physics, Royal Swedish Acad. of Sciences, SE-106 91 Stockholm, Sweden

Axel Brandenburg

NORDITA, Blegdamsvej 17, DK-2100 Copenhagen Ø, Denmark

February 7, 2020

\begin{abstract}
Statistical parameters of the ISM driven by thermal energy injections from supernova explosions have been obtained from 3D, nonlinear, magnetohydrodynamic, shearing-box simulations for spiral arm and interarm regions. The density scale height obtained for the interarm regions is $50 \%$ larger than within the spiral arms because of the higher gas temperature. The filling factor of the hot gas is also significantly larger between the arms and depends sensitively on magnetic field strength.
\end{abstract}

Keywords: ISM, turbulence, galactic spiral structure

Detailed observations of the multi-phase interstellar medium (ISM) have recently been supplemented with extensive numerical simulations that capture much of the physics involved, including driving by supernovae (SNe) and radiative cooling. Several of the models are in 3D (e.g., de Avillez, 2000;de Avillez and Berry, 2001; de Avillez and Mac Low, 2001), but only that of Korpi et al. (1999a,b) includes rotation and so is suitable for realistic modelling of magnetic fields. Here we discuss a further development of this model, where we concentrate on the effects of the total gas column density and magnetic field strength on the properties of the ISM.

Our models for regions inside and outside the spiral arms differ in the gas column density; the midplane density $\rho_{0}$ is given in Table I. The three models with low, intermediate and high densities are referred to as Interarm, Average and Arm. The column density does not vary in time in our simulations. Thus, our model is applicable not far from the corotation radius of the spiral pattern, and so is complementary to the model of Gómez \& Cox (2002) which focuses on dynamic effects due to the passage of a density wave through the ISM.

We solve numerically the 3D, non-ideal MHD equations with rotation, density stratification in external gravity, heat sources and ra-

(C) 2020 Kluwer Academic Publishers. Printed in the Netherlands. 
Table I. Three models with varying initial mid-plane density, $\rho_{0}$. The mean temperature, pressures, root mean square velocity and filling factor are all calculated within $|z|<0.2 \mathrm{kpc}$. The filling factors are for $T>10^{5} \mathrm{~K}$; the initial magnetic field at the midplane is $B_{0}=6 \mu \mathrm{G}$ in all models.

\begin{tabular}{lcccc}
\hline & Unit & Interarm & Average & Arm \\
Initial midplane density, $\rho_{0}$ & $10^{-24} \mathrm{~g} \mathrm{~cm}^{-3}$ & 0.7 & 1.4 & 2.9 \\
\hline Density Gaussian scale height & $\mathrm{kpc}$ & 0.23 & 0.20 & 0.16 \\
Mean temperature & $10^{4} \mathrm{~K}$ & 35 & 7.8 & 3.4 \\
Mean thermal pressure & $10^{-14} \mathrm{dyn} \mathrm{cm}^{-2}$ & 42 & 68 & 120 \\
Rms vertical velocity & $\mathrm{km} \mathrm{s}^{-1}$ & 23 & 20 & 20 \\
Mean turbulent pressure & $10^{-14} \mathrm{dyn} \mathrm{cm}^{-2}$ & 39 & 63 & 110 \\
Hot gas filling factor & & 0.12 & 0.07 & 0.04 \\
SN II rate & $\mathrm{kpc}^{-2} \mathrm{Myr}^{-1}$ & 11 & 38 & 111 \\
SN II Gaussian scale height & $\mathrm{kpc}$ & 0.30 & 0.16 & 0.14 \\
\hline
\end{tabular}

diative cooling (Korpi et al., 1999a). The simulations start with an imposed azimuthal magnetic field of variable strength $B_{0}$, with vertical profile $B_{0} \cosh ^{-2}(z / 0.3 \mathrm{kpc})$. The system is driven by localised thermal energy injections, modelling SN explosions. Type II SNe are initiated at those locations where the gas density $\rho$ exceeds $\rho_{\mathrm{c}}=10^{-24} \mathrm{~g} \mathrm{~cm}^{-3}$ and temperature $T$ is lower than $T_{\mathrm{c}}=4000 \mathrm{~K}$; the probability of an SN explosion at a given position is proportional to the local gas density within those regions. The SN II rate, $\nu_{\mathrm{SN}}$ II, depends on the local ISM parameters via (Gudiksen, 1999)

$$
\nu_{\mathrm{SN} I I}=\frac{M_{\mathrm{c}} X_{\mathrm{SN}} X_{*}}{M_{\mathrm{SN}} \tau_{\mathrm{c}}}
$$

where $M_{\mathrm{c}}$ is the mass of gas where $\rho>\rho_{\mathrm{c}}$ and $T<T_{\mathrm{c}}, X_{\mathrm{SN}}=0.1$ is the fraction of the stellar mass in $\mathrm{SN}$ progenitors obtained from a suitable initial mass function, $X_{*}=0.02$ is the gas mass fraction converted into stars, $M_{\mathrm{SN}}=10 M_{\odot}$ is the SN progenitor mass, and $\tau_{\mathrm{c}}=$ $20 \mathrm{Myr}$ is the gas recycling time. The latter has been adjusted to have $\nu_{\mathrm{SN} \text { II }} \simeq 40 \mathrm{kpc}^{-2} \mathrm{Myr}^{-1}$ (1/33 yr for the whole Galaxy) for the average gas density; we stress that both the rate and spatial distribution of Type II SNe are not prescribed but rather follow from the simulations. The resulting dependence of the SN II rate on the mean gas density, shown in Fig. 1, is consistent with the observed scaling of the star formation rate with gas surface density, SFR $\propto \Sigma^{\kappa}$ with $\kappa=0.9-1.7$ (Kennicutt, 1998). The SN II rates and scale heights are given in Table I. Our model also includes Type I SNe implemented as in Korpi et al. (1999a). 

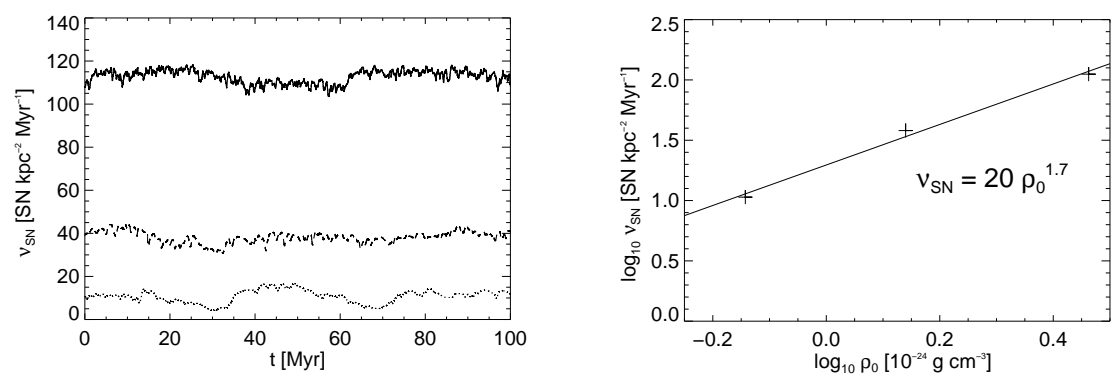

Figure 1. The SN II rate versus time (left), for the Arm (solid), Average (dashed) and Interarm (dotted) models. The scaling of the mean SN II rate with mid-plane density, $\rho_{0}$ (right).

Our results, as reported here, are preliminary because we use a relatively small computational domain with the horizontal and vertical (z) dimensions of $0.25 \times 0.25 \times 1 \mathrm{kpc}$ (with the midplane in the centre), modest spatial resolution of about $4 \mathrm{pc}$, and closed boundary conditions in $z$. Furthermore, the cooling function was truncated at $500 \mathrm{~K}$ to avoid high densities. All these restrictions are of a technical nature and can readily be relaxed.

An unexpected result of our simulations is that the density scale height is significantly larger in the Interarm model, although both thermal and turbulent pressures are a factor of about 3 larger in the Arm model. The reason is that the gas temperatures are higher in the Interarm case (Fig. 2), even though the SN rate is lower. An apparent reason is that the cooling rate has a stronger net dependence on gas density than the SN energy injection rate.
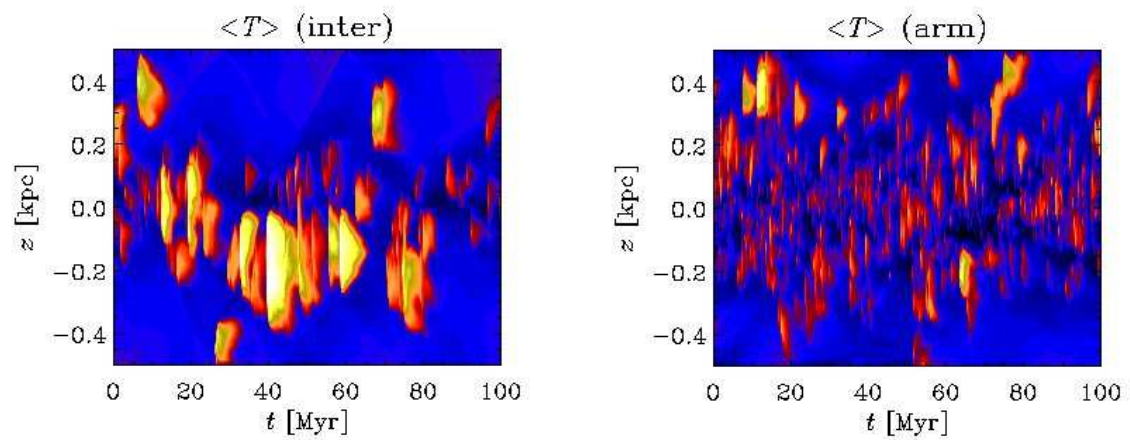

Figure 2. Horizontally averaged temperature as a function of height and time, for the Interarm (left panel) and Arm (right panel) solutions. Lighter shades of grey indicate higher temperatures. 
Table II. The effect of magnetic field on the ISM illustrated with two runs with varying initial magnetic field, $B_{0}$. All variables are as defined in Table I. All runs have $\rho_{0}=0.7 \times 10^{-24} \mathrm{~g} \mathrm{~cm}^{-3}$ (the Interarm model).

\begin{tabular}{lccc}
\hline Initial magnetic field strength, $B_{0}$ & $\mu \mathrm{G}$ & 0 & 6 \\
\hline Density scale height & $\mathrm{kpc}$ & 0.20 & 0.23 \\
Mean thermal pressure & $10^{-14} \mathrm{dyn} \mathrm{cm}^{-2}$ & 50 & 42 \\
Rms vertical velocity & $\mathrm{km} \mathrm{s}^{-1}$ & 43 & 23 \\
Mean turbulent pressure & $10^{-14} \mathrm{dyn} \mathrm{cm}^{-2}$ & 54 & 39 \\
Hot gas filling factor & & 0.19 & 0.12 \\
\hline
\end{tabular}

Another surprising feature of the results presented in Table I is that the filling factor of the hot gas is lower than expected by a factor of $2-3$. This can be attributed to the geometry of the magnetic field in our models; it is uniform initially, and therefore effective in confining expanding bubbles of hot gas. The initial midplane field strength, $6 \mu \mathrm{G}$, is close to that of the total field in the Solar vicinity, but the field is implausibly well ordered. A more realistic simulation would initialize the model with a ratio of turbulent to ordered magnetic energy of about 3. We postpone such calculations for future work, but here we explore the dependence of the results on the strength of the initially uniform magnetic field; the results are shown in Table II. The filling factor of the hot gas is sensitive to the field strength and increases to 0.2 as the field becomes weaker. The density scale height marginally increases with magnetic field strength, but this effect is much less pronounced than the suppression of the hot phase; the field strongly suppresses turbulence in the hot gas. Thus, the magnetic field can affect the dischalo connection and the global structure of the ISM in crucial, diverse and unexpected ways. This aspect of the ISM dynamics has not yet been fully explored.

\section{Acknowledgements}

We are grateful to M.-M. Mac Low for helpful comments on the dependence of the filling factor of the hot gas on magnetic field. Use of the Copenhagen branch of the Danish Center for Scientific Computing and the PPARC supported UKAFF computer facility at Leicester is acknowledged. This work was supported by the PPARC Grant PPA/G/S/2000/00528. 


\section{References}

de Avillez, M. A. MNRAS, 315: 479, 2000.

de Avillez, M. A. and D. L. Berry MNRAS, 328: 708, 2001.

de Avillez, M. A. and M.-M. Mac Low ApJ, 551: L57, 2001.

Gómez, G. C. and D. P. Cox. ApJ, 580: 235, 2002.

Gudiksen, B. V. Master Thesis, Astron. Obs., Univ. Copenhagen, 1999.

Kennicutt, R. C. ARAA, 36: 189, 1998.

Korpi, M. J., A. Brandenburg, A. Shukurov, I. Tuominen and Å. Nordlund. ApJ, 514: L99, 1999a.

Korpi, M. J., A. Brandenburg, A. Shukurov and I. Tuominen. A\&SA, 350: 230, 1999b. 
paper.tex; 7/02/2020; 16:51; p.6 